Antibiotic Resistance in Coliforms from Animal Feeding Stufís

D. H. M. JOYNSON AND C. H. L. HOWELLS (Public Health Laboratory, University Hospital of Wales, Cardiff) Samples of animal feeding stuffs were examined for the presence of Gram-negative, lactosefermenting organisms (hereafter called coliforms) and the antibiotic resistance of any strain isolated was determined.

Coliforms were found in $13 \%$ of all the feeds examined and of these organisms $64.4 \%$ were found to be resistant to one or more antibiotic.

A high proportion $(45.5 \%)$ were resistant to ampicillin, but resistance to other antibiotics was lower: colistin (16.6\%), streptomycin $(10.6 \%)$, tetracycline $(5 \cdot 8 \%)$, and neomycin $(0 \cdot 1 \%)$.

Resistance to three or more antibiotics was demonstrated by $6.5 \%$ of the coliforms isolated. Transfer of R-factors to a sensitive $E$. coli recipient was demonstrated in $86.4 \%$ of the multiresistant strains examined.

Antibiotic-resistant strains of coliforms have been regarded as a danger to human health for many years. It is believed that these bacteria arise from animals which have been given antibiotics as growth supplements, the organisms eventually finding their way to man via untreated meats, etcetera. It has also been demonstrated that some of these microorganisms may find their way into the hospital kitchen and thus, via washing-up water and utensils, to the patients.

As far as known, however, no one has yet suggested that animal feeding stuffs already containing antibiotic-resistant strains of coliforms may be the source of animal infection with such organisms.

\section{Three Studies of Salmonellae in South} Wales

R. W. S. HARVEY (Public Health Laboratory, University Hospital of Wales, Cardiff) Salmonella surveillance has been one of our interests in Cardiff during the last twenty or more years. Three studies in monitoring this disease were described. The first investigation was performed on the incidence of salmonellae in the River Taff. Our attention was drawn to the presence of these organisms in the river, when advice was asked about the dangers of bathing in it. A wide range of serotypes was isolated in this study. Some of these were probably of human origin ( $S$. paratyphi $B, S$. typhimurium, S. panama, and
$S$. brandenburg). These were the serotypes prevalent in human infection during the river survey.

Further information was sought on the origin of the wide range of salmonella serotypes found and a survey of human sewage was. arranged. An estate housing 4000 persons was selected. Surface water samples were negative for salmonellae. Sewage samples were consistently positive. The survey when completed revealed the presence of a wide range of serotypes. Some were unusual (Subgenus II serotypes) and suggested an exotic source. The wide range of salmonella recovered could be explained by animal feed playing a significant part in the epidemiology of salmonellosis in the United Kingdom.

Poultry consume infected animal feed and the final survey was directed to the salmonella content of poultry samples entering the kitchens of the University Hospital of Wales. The samples frequently contained salmonellae and there was some correspondence between the serotypes isolated from the poultry and those found in sewer swabs placed in the paediatric department of the University Hospital of Wales during the survey period. The poultry serotypes also corresponded to some extent with those found in man in Cardiff in the same time period.

Further Studies on Giant Nuclear Masses in Disseminated Intravascular Coagulation

H. B. GOODALL (Ninewells Hospital and Medical School, Dundee) Giant nuclear masses have been found in the lungs and blood in malignant malaria (Goodall, 1973a and b). As these structures may be derived from vascular endothelial cells damaged in disseminated intravascular coagulation (DIC), further studies have been made on the blood of patients with disseminated intravascular coagulation associated with other disorders, particularly endotoxin shock, acute pancreatitis, accidental hypothermia, and obstetric complications. Simple quantitative techniques with buffy coat and thick film preparations have been used on patients and control subjects. Smears have been stained with Leishman's stain, Giemsa's stain, and the Feulgen technique.

Two questions have been asked. First, do giant nuclear masses commonly occur in the blood of patients with disseminated intravascular coagulation? The answer is yes! Second, is the presence of such masses always a harbinger of death? The answer is no!

\section{Reference:}

Goodall, H. B. (1973a). Lancet, 2, 1124. Goodall, H. B. (1973b). J. clin. Path., 26, 982.

Absorption of Hexachlorophane from Newborn Infants' Skin

V. G. ALDER AND W. A. GILLESPIE (Bristol $\stackrel{\curvearrowright}{\overparen{Q}}$ Royal Hospital for Sick Children and 으 University of Bristol) In a maternity $\infty$ hospital in which the umbilicus and trunk $\overrightarrow{0}$ of healthy newborn infants were treated with 0.33 per cent hexachlorophane $\vec{\omega}$ dusting powder, the hexachlorophane content of blood was measured in mothers $\frac{0}{0}$ before delivery, in infants' umbilical is samples at birth, and at 8 days of age $V$ in capillary blood samples. One mother $\overrightarrow{-}$ and her baby had rather high blood levels $c$ of hexachlorophane, probably derived $\vec{\omega}$ from a toilet preparation used before ${ }^{\circ}$

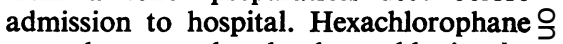
was absent or barely detectable in the other mothers' blood and in the infants' $Z$ umbilical blood. The hexachlorophane concentrations in the blood of 8-dayold infants ranged from nil to $0.166 \mu \mathrm{g}$ per ml (mean $0.066 \mu \mathrm{g}$ per $\mathrm{ml}$ ). These were much less than the concentratiogs reported to be toxic in animals.

In a previous trial now reported here a dusting powder containing chlorhexiditi instead of hexachlorophane was found to delay the separation of the umbilical cord, perhaps because of its wider antibacterial range of action. Because this delay was sometimes inconvenient, the trial of chlorhexidine was discontinued.

Efiect of Pregnancy on Calcium Homeo stasis in Asian Immigrants

NINA POLANSKA, R. A. DALE, AND M. R. WILLs (Departments of ChemicalPathology, West Middlesex Hospital and Royal Free Hospital, London) It is recognized that rickets in children and osteomalacia in adults, both biochemical and clinical, occur more frequently in Asian immigrants to this country than in the indigenous white population.

During pregnancy, because of the $N$ increased demand for calcium for growth of the fetal skeleton, a negative calcium balance may occur in the mother particularly if she is already vitamin $D$ deficient. It is recognized that during the progress of normal pregnancy there is a variation in the concentration of many plasma constituents with, in particular, a reduction in total protein, albumin, and calcium, and with a rise in alkaline phosphatase 
activity; the latter being due to a contribution from the placental isoenzyme fraction.

A study of Asian women attending an antenatal clinic was undertaken to observe the effect of pregnancy on calcium metabolism, and the incidence of biochemical and overt osteomalacia in such a population. Seventy-two Asian women of Indian and Pakistani origin were studied and compared with a control group of 20 Caucasian women and 15 coloured non-Asians mainly of negro origin. Random blood specimens which were collected at booking and after 34 weeks of gestation were analysed by routine methods for calcium, phosphate, alkaline phosphatase, total protein, and albumin. Each patient was questioned with regard to general health, dietary habits, and time spent out of doors.

In the groups studied calcium values fell as pregnancy progressed and in the Asians this was not accounted for by the reduction in albumin concentration which is known to occur in pregnancy.

The reduction in plasma calcium concentration in the Asians would appear to be mainly due to dietary factors combined with a reduction in endogenous cholecalciferol synthesis.

\section{Circannual Excretory Patterns in Man}

A. G. GREeN (County Hospital, York) Using groups of at least 30 , and where possible over 40 , subjects and dividing the year arbitrarily into four seasons of winter (December-February), spring (March-May), summer (June-August), and autumn (September-November) has resulted in the demonstration of significant circannual or seasonal variations in the excretion of several substances.

A total of 138 male controls divided into four groups gave the following mean values for urinary calcium excretion: winter $223 \mathrm{mg} / 24 \mathrm{~h}$, spring $256 \mathrm{mg} / 24 \mathrm{~h}$, summer $294 \mathrm{mg} / 24 \mathrm{~h}$, and autumn 261 $\mathrm{mg} / 24$ hours. A similar type of excretory pattern was found in 245 male postpartial gastrectomy patients where the extreme values were winter $123 \mathrm{mg} / 24 \mathrm{~h}$ and summer $179 \mathrm{mg} / 24$ hours. One hundred and seventy-four males suffering from duodenal ulcers exhibited a similar trend in calcium excretion although in these cases the differences were not statistically significant. These variations could not be accounted for on the basis of age, variation in dietary intake, or variations in analytical technique.

Similar circannual excretory patterns were found in the case of the cortisol metabolite tetrahydrocortisone (and also THE) and also for the adrenaline metabolites metadrenaline and normetadrenaline.

The implications of these findings are discussed in relation to investigations involving urine studies and in relation to the interpretation of clinical investigations.

A Comparative Study of a Glycoprotein Isolated from Gastric Aspirates, Normal Gastric Mucosa, and Gastric Carcinomata

J. SCHRAGER (Royal Albert Edward Infirmary, Wigan) The isolation and partial characterization of glycoproteins isolated from normal gastric aspirates, from extracts of normal gastric mucosae, and from extracts of gastric carcinomata are reported.

The carbohydrate and amino acid composition of the glycoproteins isolated from individual gastric aspirates and from extracts of individual gastric mucosae showed that a basic common carbohydrate composition, galactose/ $\mathbf{N}$-acetylglucosamine/N-acetylgalactosamine, was $4: 3: 1$.

Superimposed on this basic structure were additional sugar residues associated with blood group specificity, an additional fucose residue with blood group $\mathrm{H}$, a further galactose with $B$, and a further $\mathrm{N}$-acetylgalactosamine with $\mathrm{A}$ activity. Threonine and serine constituted $40-50 \%$ and these, together with proline, alanine, and glycine, accounted for $80-85 \%$ of the total amino acid content.

Mass spectrometry, periodate oxidation, reduction by borohydride, and $\beta$-elimination results strongly suggest that the carbohydrate side chains are composed of repeating disaccharide units, each unit being made up of a galactose and $\mathrm{N}$ acetyl hexosamine, the unit nearest to the protein core being $\mathrm{N}$-acetyl-galactosamine, which links the side chain to threonine and serine, each remaining unit consisting of a galactose and an $\mathrm{N}$-acetylglucosamine.

The glycoproteins isolated from gastric carcinomata contained the same range of sugars but revealed significant differences. (a) The quantitative relationships of the carbohydrate components of the neoplastic glycoproteins showed variations dividing the samples investigated into four groups, each group with a distinctive and constant carbohydrate composition: group 1, galactose/glucosamine/galactosamine = 1:1:1 (1 case); group 2, galactose/glucosamine/galactosamine 2:1:1 (1 case); group 3, galactose glucosamine/galactosamine $=3: 2: 1 \neq 9$ cases); group 4, galactose/glucosamine galactosamine $=4: 3: 1$ (9 cases). (b) The blood group specificity of 11 out of 20 gastric carcinomata investigated differ from that of the hosts' red cells.

\section{Renal Failure in Paraplegics}

C. R. TRIBE (Southmead Hospital, Bristö) Renal failure is by far the commonest cause of death in paraplegics who survige more than three months following the्g.r paralysis. Necropsies were performed kn 179 chronic paraplegics who died at Sto Mandeville Hospital between 1945 and 1973 from causes related to their paris. plegia. In $135(75.5 \%)$ patients, renal failure was considered tc be the primady' cause of death.

The renal sequelae of chronic paraplegia develop from two main causes. First, impairment of bladder functiô followed by catheterization leads urinary sepsis and back pressure on te kidney; these, combined with sta\$s caused by immobility, lead to calcubosis and chronic pyelonephritis. Second ${ }_{0}$ the muscular paralysis and loss of sensaifin lead to the development of pressure sirg, osteomyelitis, and a high incidence of amyloidosis; 99 out of the 135 cas dying in renal failure showed amylogid disease. Secondary hypertension is oft associated with the chronic disease.

A previously published detailed stu武 of 220 necropsies performed on paraplegics between 1945 and 1965 forms tre basis of this presentation ${ }^{1}$. The relationships of the different factors leading renal failure were discussed and descriptions of the types of pyelonephritis. amyloidosis, and hypertension in the patients were presented with tabulation of results and illustrations of the releva renal pathology. As an indication of tbe 'mixed' renal pathology it was considered that of the 75 patients dying in rent failure in whom adequate renal histe. logical tissue was available, pyelong. phritis contributed to renal failure in $95 \%$, amyloidosis in $50 \%$, and hypes tension in $43 \%$. The original literatunce should be studied to appreciate the plethora of renal disease which occurs tin paraplegic patients.

Reference

${ }^{1}$ Tribe, C. R., and Silver, J. R. (1969). Rem, Failure in Paraplegia. Pitman, London. 